\title{
Treatment of bilateral superior oblique tendon sheath syndrome complicating rheumatoid arthritis
}

\author{
MARILYN BECK ${ }^{1}$ AND PETER HICKLING ${ }^{2}$ \\ From the ${ }^{1}$ Department of Ophthalmology and ${ }^{2}$ Department of Rheumatology, \\ University Hospital of Wales, Cardiff
}

SUMmARY A patient with well established seropositive rheumatoid disease developed bilateral superior oblique tendon sheath syndromes. Treatment with local injections of corticosteroids is described.

\section{Case report}

A 63-year-old retired mining engineer with rheumatoid arthritis presented in November 1976 with intermittent diplopia on upward gaze. His diplopia became increasingly troublesome, particularly on waking, and he was admitted under the care of the rheumatologists for investigation in February 1977.

He had a 6-year history of seropositive erosive rheumatoid arthritis with numerous extra-articular features, including weight loss, subcutaneous nodules, Raynaud's phenomenon, mild bilateral carpal tunnel syndrome, nail fold vasculitis, hepatosplenomegaly and pericarditis.

\section{INVESTIGATIONS}

The differential agglutination test for rheumatoid factor was positive $1 / 1024$; antinuclear factor negative; erythrocyte sedimentation rate $42 \mathrm{~mm} / 1 \mathrm{st}$ hour; and full blood count normal, including differential white cell count. Thyroid function tests were normal and thyroid antibodies negative; tests for antismooth muscle and antimitochondrial antibodies were negative. The immunoglobulin profile was normal; serum alkaline phosphatase slightly elevated, but other liver function tests were normal. Technetium scan of liver and spleen showed a smooth uptake; liver biopsy was normal and sections stained for amyloid were negative. Lymph node biopsy showed reactive hyperplasia but no infiltrate.

OCULAR FINDINGS

In September 1977, as the diplopia was still present,

Correspondence to Mrs Marilyn Beck, University Hospital of Wales, Heath Park, Cardiff CF4 4XW. he was referred to the ophthalmology department.

On examination his corrected visual acuities were $6 / 9$ in each eye. Cover test showed a slight alternating hyperphoria. There was bilateral limitation of elevation in adduction and downshoot of each adducted eye on lateral versions. No clicking sensation was noted. A Hess chart confirmed that he had bilateral superior oblique tendon sheath syndromes (Fig. 1).

\section{PROGRESS}

It was decided to treat his rheumatoid disease with weekly injections of intramuscular gold in the hope of controlling his general condition and his diplopia. Unfortunately he developed such violent reactions to the injections in the form of rigors and joint pains that they had to be stopped after 6 weeks. Penicillamine was then cautiously introduced, but he soon developed diarrhoea and a rash, so therapy was discontinued. His rheumatoid disease was therefore controlled with indomethacin and aspirin.

As his diplopia persisted, in March 1978 he was given an orbital injection of $1 \mathrm{ml}(40 \mathrm{mg})$ of methylprednisolone acetate (Depomedrone) over the superomedial aspect of the left globe. Some lessening of his diplopia and improvement in his Hess chart were noted, and therefore a week later a similar orbital injection was given on the right side. A month later the patient was symptomatically very much improved, experiencing diplopia only on extreme upward gaze. A Hess chart recorded on this occasion is shown in Fig. 2.

Six months later his diplopia began to return and by March 1979 was no longer tolerable. Ocular examination and Hess chart (Fig. 3) showed a recurrence of his bilateral superior oblique tendon sheath syndrome. Orbital injections of $1 \mathrm{ml}$ of 
methylprednisolone acetate were given superomedially over each globe. Four weeks later he had improved greatly subjectively and objectively (Fig. 4) and remains asymptomatic 3 months after these injections.

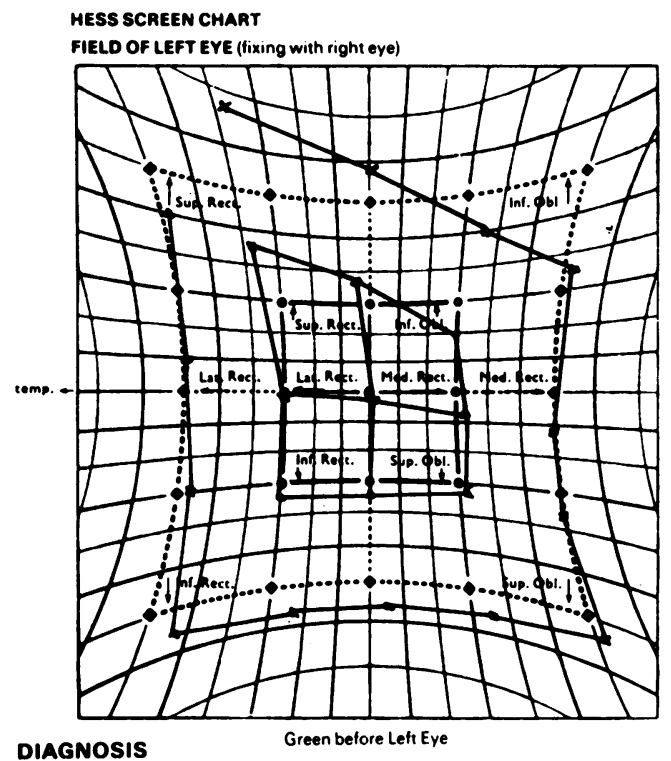

Fig. 1 Hess chart in September 1977.
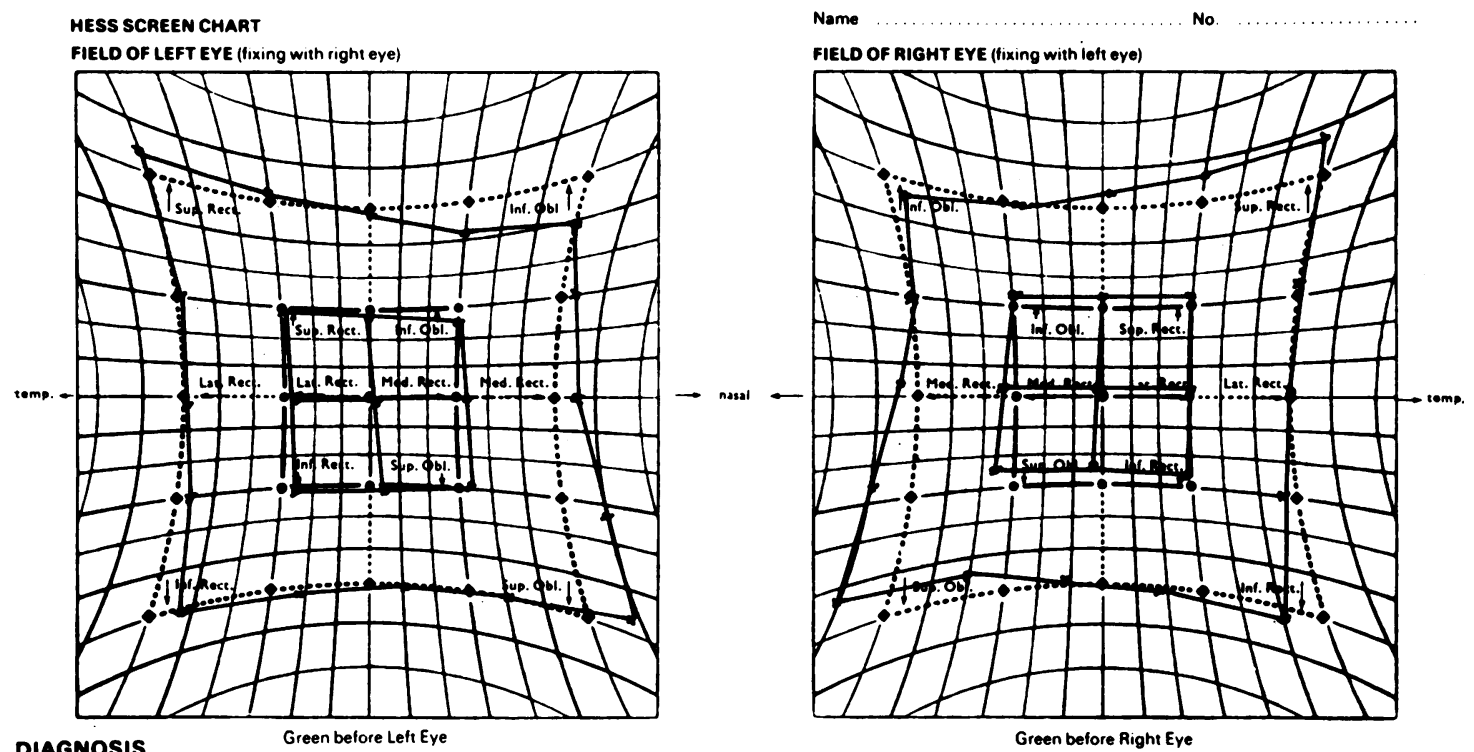

Fig. 2 Hess chart in April 1978.

\section{Discussion}

Harold Brown ${ }^{1}$ described the superior oblique tendon sheath syndrome which has since been called Brown's syndrome. The syndrome was

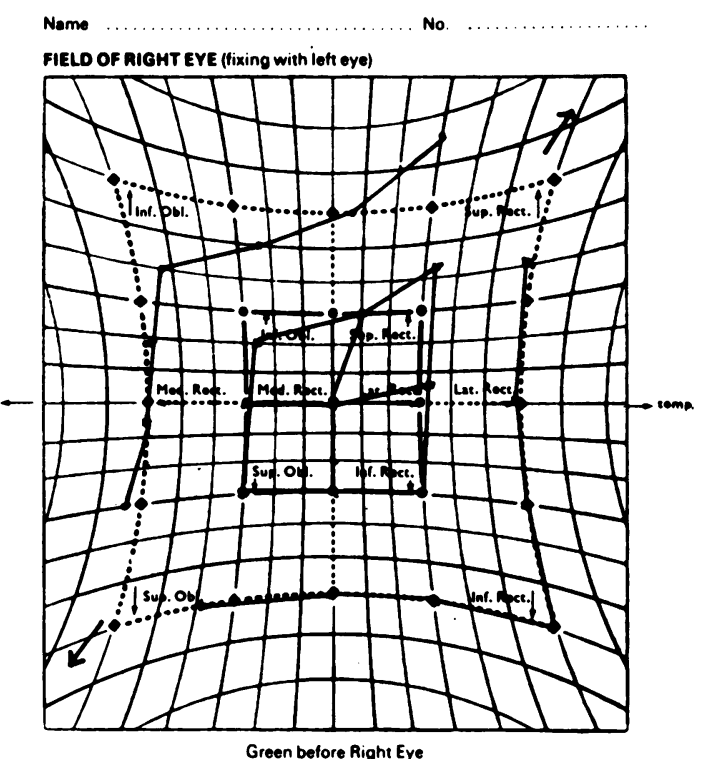


initially thought to be the result of a congenital paralysis or delay in function of the inferior oblique muscle. This was postulated to lead to a thickening and shortening of the anterior sheath of the superior oblique, which is the check ligament of the inferior oblique muscle. The various theories of the aetiology of the syndrome are well reviewed by $\mathrm{Mein}^{2}$ and Sandford-Smith. ${ }^{3}$

Brown ${ }^{4}$ expanded the description of the syndrome, separating it into true and simulated sheath syn-
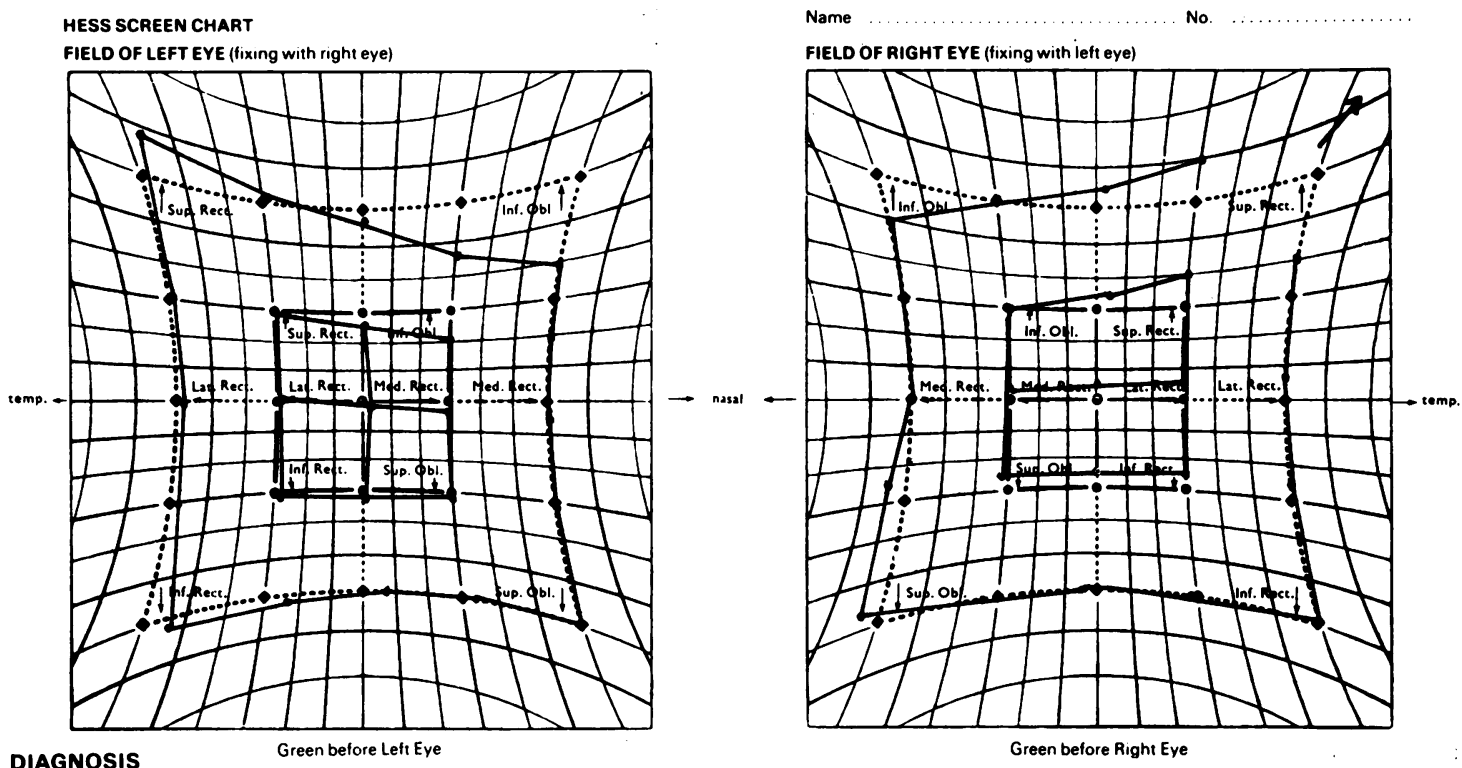

Fig. 3 Hess chart in March 1979.
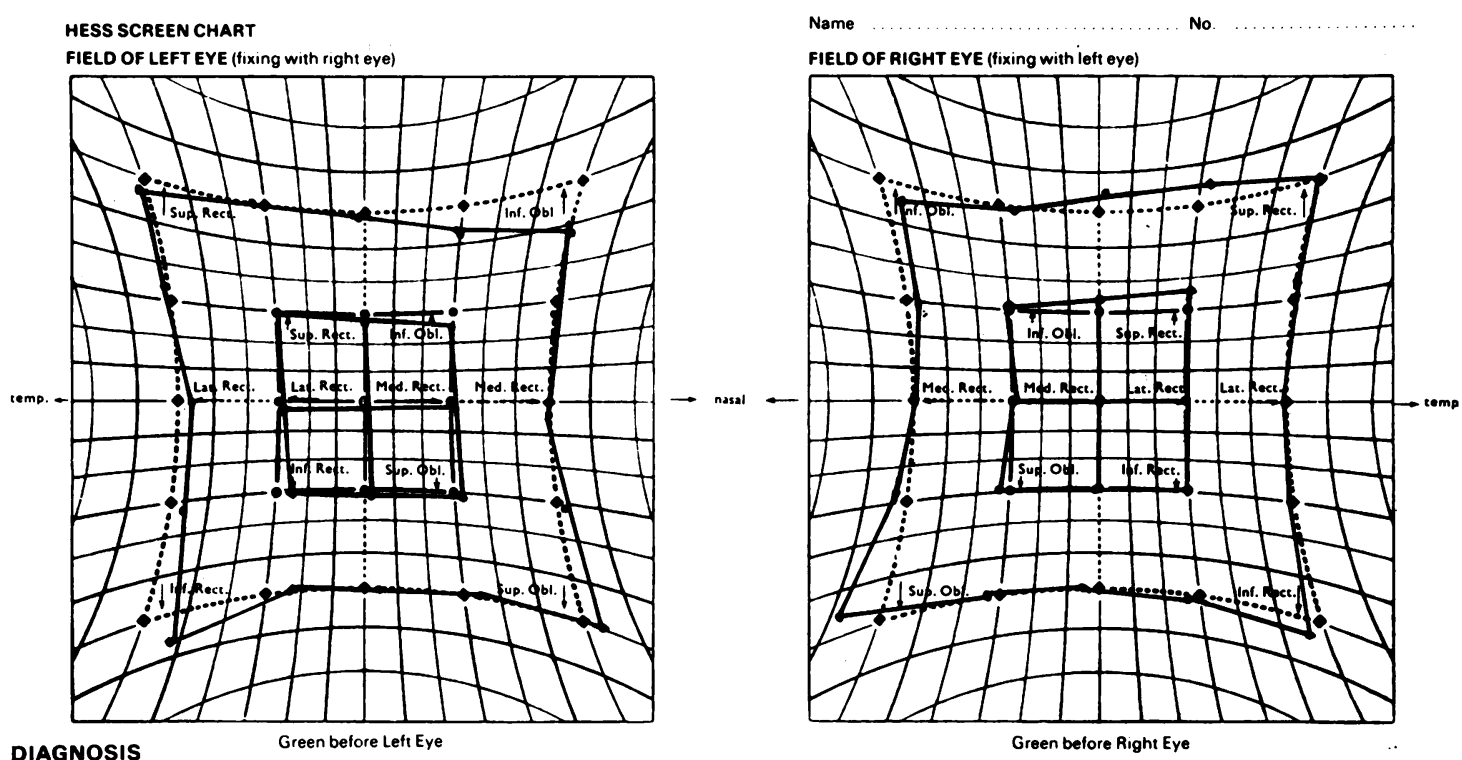

Fig. 4 Hess chart in April 1979 
dromes. The true sheath syndrome is always congenital and never disappears spontaneously. The simulated syndrome, however, may be congenital or acquired, may be intermittent and sometimes even undergo spontaneous recovery. The acquired simulated sheath syndrome has been described in association with ethmoiditis, orbital floor fractures, tucking of the superior oblique tendon, and rheumtoid arthritis.

Various surgical procedures designed to ameliorate the condition by weakening the superior oblique tendon have been reported.5 We describe the treatment of bilateral superior oblique tendon sheath syndromes with local injection of steroids in a patient with rheumatoid arthritis. As the patient improved both subjectively and objectively after each steroid injection, it seems unlikely that this was due to spontaneous recovery.

We thank Mr M V Graham and Dr George Nuki for permission to publish this case.

\section{References}

${ }^{1}$ Brown HW. Congenital structural muscle anomalies. In: Allen JM, ed. Strabismus Ophthalmic Symposium. St Louis: Mosby, 1950: 205 et seq.

${ }^{2}$ Mein J. Superior oblique tendon sheath syndrome. $\mathrm{Br}$ Orthopt J 1971; 28: 70-6.

${ }^{3}$ Sandford-Smith JH. Superior oblique tendon sheath syndrome and its relationship to stenosing tenosynovitis. Br J Ophthalmol 1973; 57: 859-65.

${ }^{4}$ Brown HW. True and simulated superior oblique tendon sheath syndromes. Doc Ophthalmol 1973; 34: 123-36.

${ }^{5}$ Parks MM. The superior oblique tendon. Trans Ophthalmol Soc UK 1977; 97: 288-304. 